MS40-05

\section{Current challenges of obtaining crystals for bio-crystallography}

Monika Spano ${ }^{1}$, Niels Junius ${ }^{1}$, Elham Vahdatahar ${ }^{1}$, Sofia Jaho ${ }^{1}$, Christophe Berzin², Yoann Sallaz-Damaz ${ }^{3}$, David Cobessi ${ }^{3}$, Jean-Luc Ferrer $^{2}$

1. Université Grenoble Alpes, Institut de Biologie Structurale, Grenoble, France

2. CEA, Institut de Biologie Structurale, Grenoble, France

3. CNRS, Institut de Biologie Structurale, Grenoble, France

email: monika.spano@ibs.fr

A rational way to find the proper conditions to grow crystal samples for Bio-Crystallography is to determine the crystallization phase diagram, which allows a punctual control of the parameters affecting the crystal growth process. In particular, the aim is to induce the nucleation at supersaturated conditions close to the solubility boundary between labile and metastable regions, by modulation of specific physical parameters. The detailed knowledge of the phase diagram is at the basis of the devices we have developed especially with the focus on Neutron Macromolecular Crystallography. The $1^{\text {st }}$ generation instrument combines the use of temperature control and seeding and allows for grow of large crystals in crystallization batch. A crystallization batch in the metastable zone is seeded with small protein crystals. The seeds are maintained inside this region of the phase diagram for as long as possible by doing a temperature step each time the crystal solution equilibrium is achieved. The temperature steps are repeated until crystals of suitable size for diffraction measurement are obtained. The $2^{\text {nd }}$ generation instrument adds new functionality to the first instrument thanks to a fluidic cell enabling to perform a temperature controlled dialysis crystallization experiment. The new crystal growth apparatus combines accurate temperature control with control of the chemical composition of the crystallization solution and therefore it allows very sophisticated experiments to be performed. Systematic phase diagrams in multi-dimensional space can be investigated using far less protein material than previously. We have demonstrated that it can be beneficial to provide sufficient scattering volumes for neutron studies that require large-volume well-ordered single crystals. Based on this macro-scale instrument we have also conceived a miniaturizing apparatus that allows precise control of the experiment parameters using microfluidics. The functional microfluidic chips integrating microdialysis with the volume less than $1 \mu \mathrm{L}$ have been already successfully tested with model proteins. The microchips have multiple designs in order to achieve single or multiple crystallization experiments at the same time. They are transparent to X-ray radiation and allow performing in situ $\mathrm{X}$-ray crystallography experiments at room temperature. The automated microfluidic pipeline we are setting up is expected to be useful in monitoring and controlling the crystallization processes of challenging biological macromolecules, such as membrane proteins. Preliminary experiments also show potential benefit for synchrotron serial crystallography that require uniformly-sized populations of microcrystals (10-20 $\mu \mathrm{m})$, optimal for time-resolved serial crystallographic experiments.
References:

[1] Budayova-Spano M. et al. (2007) Acta Cryst. D63; 339-347.

[2] Junius N. et al. (2016) J. Applied Cryst. 49, 806-813.

[3] Budayova-Spano M. et al. (2016) Patent FR2016/053185.

Keywords: neutron macromolecular crystallography, control of crystal size, temperature-controlled (micro)dialysis crystallization 